\title{
Prevalencia de enfermedad carotídea posterior a accidente cerebrovascular en un hospital universitario de Bogotá (Colombia)
} Prevalence of Carotid Disease after Stroke in a Teaching Hospital of Bogotá, Colombia

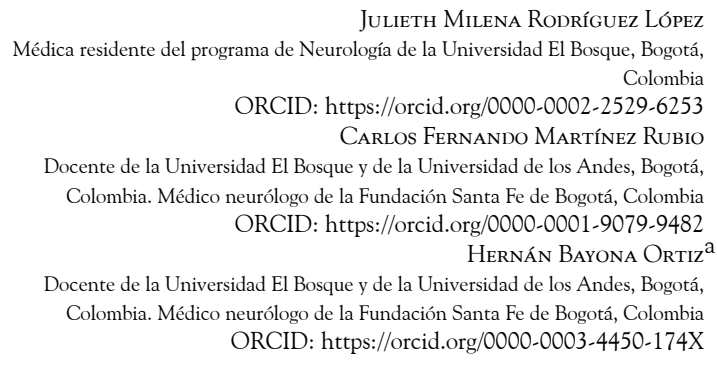

a Autor de correspondencia: hernan.bayona@fsfb.org.co

Cómo citar: Rodríguez López M, Martínez Rubio CF, Bayona Ortiz H Prevalencia de enfermedad carotídea posterior a accidente cerebrovascular en un hospital universitario de Bogotá (Colombia). Univ. Med. 2021;62(1). https://doi.org/10.11144/Javeriana. umed62-1.cacv

\section{RESUMEN}

Objetivo: Establecer la prevalencia de estenosis carotídea ( $\geq 50 \%$ ) en una población con ictus isquémico entre 2014 y 2018 y comparar con datos previos disponibles. Métodos: Se realizó un estudio de tipo corte transversal. Se incluyeron pacientes mayores de 18 años con ictus isquémico agudo, en quienes en la evaluación etiológica se documentó una estenosis carotídea $(\geq 50 \%)$, con descripción de sus principales factores de riesgo, estudios e intervenciones. Resultados: De 413 pacientes con ictus, se encontraron 51 con estenosis carotídea ( $>50$ $\%)$, con prevalencia del 12,3\%. La prevalencia previa de 1996 a 2002 fue del $16 \%$. La edad media fue de 78 años, con ligera predominancia en hombres $(51 \%)$. Dentro de los factores de riesgo hubo mayor proporción de hipertensión arterial (76,5\%) y de dislipidemia (47\%). En 15/51 se pudo revisar el acuerdo entre los métodos de imágenes diagnósticas, con un $54 \%$ de concordancia para el grado de estenosis. En el $59 \%$ de los sujetos, la clasificación etiológica del accidente cerebrovascular se debió a enfermedad de vaso grande, con un pequeño porcentaje de cirugías (12 $\%)$. Conclusiones: Se encontró una prevalencia de estenosis carotídea similar a otros estudios, con una reducción en los factores de riesgo, como el cigarrillo y menos marcadores de ateroesclerosis y menos enfermedad coronaria.

Palabras clave

accidente cerebrovascular; prevalencia; estenosis carotídea; factores de riesgo.

\begin{abstract}
Objective: To establish the prevalence of carotid stenosis $(\geq 50 \%)$ in a population with stroke between the years 2014 to 2018 and to compare it with the previous data. Methods: A cross-sectional study was performed. Patients older than 18 years with acute ischemic stroke were included, who in the etiological evaluation documented a significant carotid stenosis $(\geq 50 \%)$, with a description of their main risk factors, studies
\end{abstract}


and interventions. Results: Of 413 patients with stroke, 51 patients with stenosis $>50 \%$ were found with a prevalence of $12.3 \%$. The former prevalence between 1996-2002 was $16 \%$. Mean age was 78 years with men (51\%). Among the risk factors, there was a higher proportion of arterial hypertension (76.5\%), followed by dyslipidemia (47\%). In $15 / 51$ of the cases it was possible to check the agreement in the diagnostic imaging tests, with a concordance of $54 \%$ to qualify the stenosis grade. In $59 \%$ of the subjects the etiologic classification of the stroke was due to great vessel disease, with a small percentage of surgeries (12\%). Conclusions: A similar prevalence of carotid stenosis was found regarding previous literature, with the decreased in risk factors like smoking and less atherosclerotic markers and lower coronary artery disease.

Keywords

cerebrovascular accident; prevalence; carotid stenosis; risk factors.

\section{Introducción}

El accidente cerebrovascular (ACV) es una importante causa de morbilidad, ya que representa la quinta causa más común de muerte en Estados Unidos. Se estima que 795.000 pacientes sufren de ictus anualmente y su prevalencia incrementa con la edad (1). En estudios de poblaciones latinoamericanas se ha informado discapacidad, de moderada a severa, en el $52 \%$ de los pacientes que sobreviven al ACV, en Colombia es la primera causa de discapacidad en adultos y la tercera causa de muerte $(2,3)$.

Dentro de las principales etiologías del ACV se encuentra la estenosis carotídea, con un riesgo anual del $2 \%$ al $5 \%$ y prevalencias que ascienden al $12,5 \%$ en estenosis moderada (50 \%-69\%) en mayores de 70 años, más frecuente en los hombres (4). Dentro de los factores de riesgo de enfermedad carotídea se encuentran tabaquismo, aumento de las lipoproteínas de baja densidad (LDL), diabetes mellitus e hipertensión. $\mathrm{La}$ ateroesclerosis es un proceso patológico, multifocal e inflamatorio de las arterias, mediado por lípidos con formación de placas de ateroma en la íntima de las arterias que progresivamente afecta la luz de los vasos en diferentes territorios vasculares $(5,6)$. Las placas se tornan inestables, y al romperse, inducen la formación de trombos con liberación de factores procoagulantes hacia la circulación. También se puede presentar con el mecanismo de oclusión proximal con reducción de flujo sanguíneo, que resulta en infartos por bajo gasto en áreas limítrofes del cerebro $(6,7)$.

La severidad de la estenosis carotídea se define según los criterios del North American Symptomatic Carotid Endarterectomy Trial (Nascet), correspondiente a la evaluación mediante ultrasonografía y otros métodos de medición indirectos, como la angiotomografía (angio-TAC) o la angiorresonancia (angio-RM) $(8,9,10,11)$. De acuerdo con el grado se presenta como una estenosis moderada (50\%-69 $\%)$, estenosis severa (70\%-99\%) y de oclusión (100\%). Dependiendo del grado de estenosis y el haber presentado síntomas, se determina el riesgo de $\mathrm{ACV}$, que puede llegar a ser del 26 $\%$ en enfermedad severa; mientras que para enfermedad asintomática es de hasta un 3,3\%, anualmente $(9,12)$.

Se desconocen cifras precisas de la prevalencia de la enfermedad carotídea en América Latina - y específicamente en Colombia-, factores de riesgo e intervenciones. Este trabajo se propuso establecer la prevalencia de la estenosis carotídea significativa (mayor al $50 \%$ ) en una población con ACV isquémico entre 2014 y 2018. Como complemento, se planteó un análisis comparativo con datos previos disponibles del centro de ACV de 1996 a 2002, así como con estudios previos publicados.

\section{Métodos}

Este fue un estudio descriptivo de corte transversal para establecer la prevalencia de estenosis carotídea significativa en pacientes con ACV en el periodo 2014-2018 en un hospital universitario en Bogotá (Colombia). Se incluyeron pacientes mayores de 18 años con diagnóstico de ACV isquémico agudo con confirmación de estenosis carotídea a través de Doppler, angio-TAC o angio-RM y se excluyeron aquellos con seguimiento o estudios incompletos y aquellos hospitalizados por otra condición no neurológica que hayan cursado con ACV isquémico durante su estancia hospitalaria. Se 
tomaron el total de sujetos en los que se registró ACV isquémico en el periodo descrito.

Se extrajeron los datos de interés a través de la historia clínica del registro total de pacientes atendidos. Se describieron las características sociodemográficas y clínicas, como edad, género, severidad mediante la Escala de Accidente Cerebrovascular del National Institute of Health (NIHSS, por sus siglas en inglés), así como los factores de riesgo - por ejemplo, hipertensión arterial (HTA), dislipidemia, enfermedad coronaria, diabetes mellitus y tabaquismo-. Se detallaron el método imagenológico diagnóstico de la enfermedad carotídea (Doppler de vasos de cuello, angio-TAC o angio-RM) y cuáles pacientes fueron llevados a intervención con endarterectomía o angioplastia más colocación de endoprótesis vasculares (stent). Por último, se describieron las variables con frecuencias y porcentajes para aquellas cualitativas; y promedios, medianas, desviación estándar (DE) y rangos intercuartílicos (IQR) para aquellas variables cuantitativas. Los análisis se realizaron utilizando la herramienta estadística STATA, versión 14.2.

Dos investigadores senior (CM y $\mathrm{HB})$ supervisaron la revisión de historias clínicas, con el propósito de garantizar la calidad de la información incluida en las bases de datos electrónicas. Se tomaron historias clínicas de forma aleatoria para garantizar la veracidad de los datos. El estudio fue aprobado por el Comité Corporativo de Ética en Investigación (aprobación CCEI 11814-2020), por tratarse de un estudio con riesgo menor que el mínimo, de acuerdo con la reglamentación colombiana. El presente trabajo fue exento de consentimiento informado. La investigación se basa en las normas éticas en investigación de Helsinki; además, se protegió la identidad de los sujetos en el manejo de los datos, donde no hay forma de poderlos identificar.

\section{Resultados}

Un total de 544 pacientes cursaron con ACV isquémico, de los cuales por criterios se excluyeron 131 (52 remitidos, 18 con estudios incompletos y 61 con evento isquémico cerebral intrahospitalario). Así, se contó con una población total de 413 pacientes y 51 sujetos se diagnosticaron con estenosis carotídea significativa. Se calculó una prevalencia de estenosis carotídea significativa del 12,3\%. Sus características demográficas y clínicas se describen en la tabla 1.

\section{Tabla 1}

Datos clínicos y demográficos

\begin{tabular}{|l|r|}
\hline \multicolumn{1}{|c|}{ Características } & Total (n) $=\mathbf{5 1}(\%)$ \\
\hline Edad media (años)-DE & $78,7 \pm 10,4$ \\
Edad mínima & 57 \\
Edad máxima & 99 \\
\hline Sexo & \\
Masculino & $26(51)$ \\
Femenino & $25(49)$ \\
\hline Índice de masa corporal & \\
(kg/ $\left.\mathbf{m}^{2}\right)$ & \\
Sobrepeso & $20(39)$ \\
Obesidad & $3(6)$ \\
\hline Hipertensión arterial & $39(76,5)$ \\
\hline Dislipidemia & $24(47)$ \\
\hline Diabetes mellitus tipo 2 & \\
HbA1C $>6,5 \%$ & $10(20)$ \\
\hline Enfermedad coronaria & $12(23,5)$ \\
\hline Enfermedad renal crónica & $8(16)$ \\
\hline ACV previo & $21(41,2)$ \\
\hline Medicamentos previos & \\
Antiagregación & $28(55)$ \\
Anticoagulación & $4(8)$ \\
Estatinas & $34(67)$ \\
\hline Tabaquismo & \\
Activo & $8(16)$ \\
No activo & $9(18)$ \\
\hline Perfil lipídico & $10(20)$ \\
CT $>200$ mg/dL & $11(23,4)$ \\
LDL > 130 mg/dL & $29(62)$ \\
HDL < 40 mg/dL & $8(16)$ \\
TG $>150$ mg/dL & \\
\hline
\end{tabular}

DE: desviación estándar; $\mathrm{HbA} 1 \mathrm{C}$ : hemoglobina glucosilada; CT: colesterol total; LDL: colesterol de baja densidad; HDL: colesterol de alta densidad; TG: triglicéridos.

La edad mínima documentada fue de 57 años, con una máxima de 99 años y edad media de 78,7 años; un $90 \%$ son mayores de 65 años, y 26 sujetos correspondieron a hombres (51\%). Los factores de riesgo más frecuentes detectados fueron HTA (76,5\%) y dislipidemia (47\%). La 
Julieth Milena Rodríguez López, Carlos Fernando Martínez Rubio, Hernán Bayona Ortiz.

puntuación media de la NIHSS fue de 7 puntos, con una mayor afectación de la cerebral media derecha (45\%). En cuanto a las terapias de reperfusión empleadas, el $20 \%$ de los pacientes recibió terapia con trombólisis endovenosa, y un $8 \%$, trombectomía mecánica. Los estudios complementarios metabólicos se encuentran en la tabla 1.

La estenosis carotídea se estableció en su mayoría a través de angio-TAC, seguida por Doppler de vasos de cuello, donde cerca del 50 $\%$ de los pacientes se clasificaron en el grupo de estenosis moderadas (50\%-69\%) (tabla 2). Se realizaron ambos estudios complementarios a 15 pacientes, con los cuales se determinó el grado de estenosis carotídea a través de las pruebas diagnósticas (método Nascet), donde en un $54 \%$ hubo acuerdo (figura 1). Se clasificaron etiológicamente los pacientes según la ASCOD (13), a fin de establecer la relación de mayor probabilidad enfermedad de vaso grande como causa potencial del ACV. Así, se encontró que en 30/51 pacientes (59\%) se estableció como causa potencial (figura 2).
Tabla 2

Características de accidente cerebrovascular e intervenciones

\begin{tabular}{|l|r|}
\hline Características & Total $(\mathbf{n})=\mathbf{5 1}(\%)$ \\
\hline NIHSS ingreso (media-DE) & $7( \pm 5,8)$ \\
\hline Territorio vascular & $23(45)$ \\
CMD & $13(25,5)$ \\
CMI & $8(16)$ \\
CPI & $4(8)$ \\
CPD & $2(4)$ \\
CAI & $1(2)$ \\
CAD & \\
\hline Grado de estenosis carotídea, & \\
según (\%) & $25(49)$ \\
50-69 & $18(35)$ \\
$70-99$ & $8(16)$ \\
100 & \\
\hline Método de estudio & $26(51)$ \\
Doppler & $40(78)$ \\
Angio-TAC & $15(30)$ \\
Doppler + angio-TAC & \\
\hline Intervenciones & $10(20)$ \\
Trombólisis & $4(8)$ \\
Trombectomía & $5(10)$ \\
Endarterectomía & $1(2)$ \\
Angioplastia + endoprótesis & \\
vascular & $4(8)$ \\
\hline Muertes &
\end{tabular}

CMD: cerebral media derecha; CMI: cerebral media izquierda; CPI: cerebral posterior izquierda; CPD: cerebral posterior derecha; CAI: cerebral anterior izquierda; CAD: cerebral anterior derecha; Nascet: North American Symtomatic Carotid Endarterectomy Trial.

\section{Figura 1}

Acuerdos por Doppler y angiotomografía del grado de estenosis según los criterios del North American Symtomatic Carotid Endarterectomy Trial

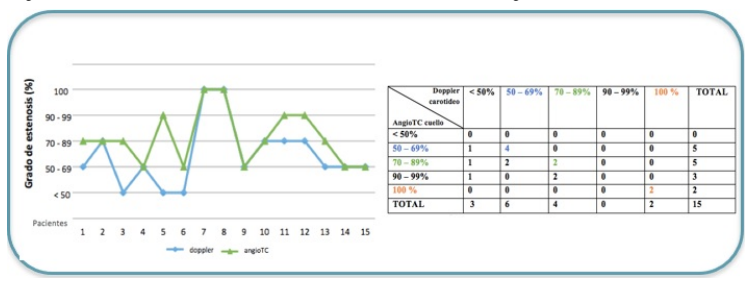




\section{Figura 2}

Representación gráfica de la clasificación ASCOD (etiología de accidente cerebrovascular)

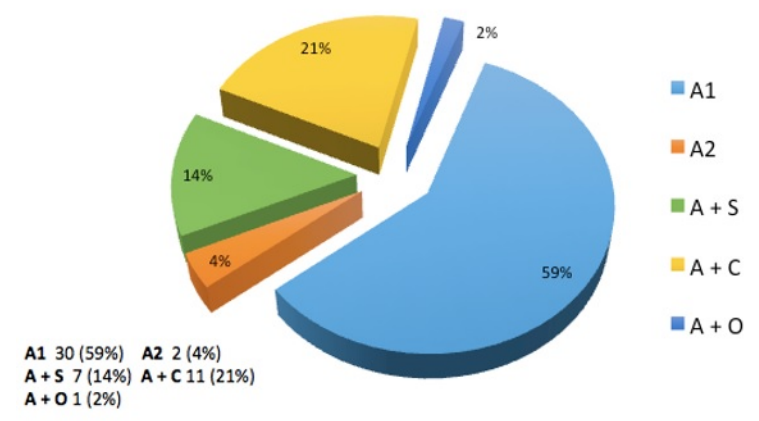

$$
\begin{gathered}
\text { A1: ateroesclerosis (causa potencial); A2: } \\
\text { ateroesclerosis causa asociada; } \mathrm{A}+\mathrm{S}: \\
\text { ateroesclerosis }+ \text { enfermedad de pequeño } \\
\text { vaso; } \mathrm{A}+\mathrm{C} \text { : ateroesclerosis }+ \text { patología } \\
\text { cardiaca; } \mathrm{A}+\mathrm{O} \text { : ateroesclerosis }+ \text { otras causas. }
\end{gathered}
$$

Solo un $12 \%$ de la población fue llevada a intervención quirúrgica o endovascular para el manejo de la estenosis carotídea. El $10 \%$ de los pacientes fue llevado a endarterectomía, y un $2 \%$, a angioplastia más endoprótesis vascular (tabla 2), de los cuales todos ellos se encontraban en la categoría de estenosis severa (70\%-99\%). La mortalidad se registró en un $8 \%$ a causa del evento cerebrovascular.

\section{Discusión}

Entre la población de estudio se documentó una prevalencia de estenosis carotídea del 12,3\%, similar a la descrita en otros estudios $(14,15$, $16,17)$, con diferencias en la prevalencia de la enfermedad para los hombres, que fue más baja en la población del hospital, la cual solo muestra un ligero aumento para el género masculino (51 \%) (18).

Se compararon los resultados del periodo 2014-2018 $(\mathrm{n}=413)$ con datos disponibles de 1996 a 2002 (n = 298) del mismo hospital (19). En este periodo se informó una prevalencia de estenosis carotídea del $16 \%$ (19). Las características del estudio se muestran en la tabla 3. Al comparar los datos, la edad media de 78 años fue similar a la previa de 81 años (19), pero ambas son mayores a las descritas en otros trabajos de 66 a 72 años $(11,15,20,21,22)$. Existen asociaciones bien estudiadas con mayor significancia para estenosis carotídea, como son la edad, sobre todo en mayores de 70 años $(\mathrm{OR}=$ $4,68)$; género masculino $(\mathrm{OR}=2,16)$; aumento de las LDL $(\mathrm{OR}=1,84)$; HTA $(\mathrm{OR}=3,16)$, y tabaquismo $(\mathrm{OR}=6,18)(18,22,23,24,25)$.

Tabla 3

Datos comparativos con el estudio previo (1996-2002) en la Fundación Santa Fe de Bogotá

\begin{tabular}{|l|r|r|}
\hline & \multicolumn{1}{|c|}{ 1996-2002 } & \multicolumn{1}{|c|}{ 2014-2018 } \\
\hline Número de pacientes & 298 & 413 \\
\hline Edad (media) & 81 & 78,7 \\
\hline Sexo (hombres) & $25(52 \%)$ & $26(51 \%)$ \\
\hline Estenosis carotídea & $\mathbf{4 8 ( 1 6 \% )}$ & $\mathbf{5 1 ( 1 2 , 3 \% )}$ \\
\hline Hipertensión arterial & $31(64,5 \%)$ & $39(76,5 \%)$ \\
\hline Dislipidemia & $27(56,2 \%)$ & $24(47 \%)$ \\
\hline Diabetes mellitus tipo 2 & $12(25 \%)$ & $10(20 \%)$ \\
\hline Enfermedad coronaria & $12(25 \%)$ & $12(23,5 \%)$ \\
\hline Tabaquismo & $18(37,5 \%)$ & $17(34 \%)$ \\
\hline
\end{tabular}

También se han asociado la diabetes mellitus tipo 2 y la enfermedad coronaria como condicionante de enfermedad carotídea severa $(14,19,22)$. En estas dos mediciones, en diferentes periodos (1996-2002 vs. 2014-2018), los factores de riesgo se encuentran presentes y en proporciones muy similares; sin embargo, hay mayor proporción de HTA y de dislipidemia para los datos actuales, con menor proporción de enfermedad coronaria, diabetes y tabaquismo respecto a otras poblaciones como la del estudio Nascet $(9,26,27)$.

Se ha descrito la hipercolesterolemia como factor de riesgo de progresión del grado de estenosis carotídea, tasa que aumenta con estenosis $>50 \%$, con $\mathrm{OR}=2,2$. Para la población actual se encontró un LDL elevado (>130 $\mathrm{mg} / \mathrm{dL})$ en un $23,4 \%$, factor que debe siempre intervenirse, dado que la disminución en las concentraciones de LDL $<70 \mathrm{mg} / \mathrm{dL}$ pueden retrasar la progresión de la enfermedad ateroesclerótica $(7,28)$. Como dato importante, se encontró que un $67 \%$ de los pacientes venía recibiendo estatinas previo al ACV (tabla 1). También son factores asociados de mayor riesgo de progresión el mal control de la presión arterial diastólica, diabetes mellitus, tabaquismo, 
Julieth Milena Rodríguez López, Carlos Fernando Martínez Rubio, Hernán Bayona Ortiz.

ser hombre (5) y el antecedente ACV o ataque isquémico transitorio.

En el presente estudio se evaluó, a través de la clasificación ASCOD, la relación causal entre ACV con ateroesclerosis como principal etiología. De nuestra cohorte de pacientes a quienes se les documentó estenosis carotídea extracraneal, el $59 \%$ fue clasificada dentro del grupo de ateroesclerosis como causa potencial; el $21 \%$, con enfermedad carotídea significativa en pacientes con antecedentes o hallazgos de novo de fibrilación auricular, y un $14 \%$, con asociación de enfermedad carotídea con enfermedad de pequeño vaso (figura 2 ).

Barnett et al. (25) encontraron en un estudio de 2885 pacientes ACV catalogados como cardioembólicos en territorio de arteria carótida sintomática en un $19 \%$ para estenosis entre el $60 \%$ y el $99 \%$. En la población estudiada se encontró esta asociación en proporciones similares.

La enfermedad ateroesclerótica carotídea puede controlarse con el mejor tratamiento médico, así como con control de factores de riesgo, con modificación de estilo de vida e intervención quirúrgica o percutánea $(7,10)$. Se ha demostrado el beneficio de estas intervenciones, principalmente en aquellos pacientes con severidad de las estenosis entre el $70 \%$ y el $99 \%(5,7,8)$.

Los datos sugieren una reducción del riesgo de recurrencia de ACV o ataque isquémico transitorio en pacientes con mayor grado de estenosis llevados a intervención, con reducción absoluta del riesgo $(17 \%)$ en pacientes con estenosis del $70 \%$ al $99 \%$, por lo cual la gravedad de la estenosis es un parámetro para la selección del tratamiento $(7,8,21)$.

En la población descrita, a 18/51 pacientes $(35,3 \%)$ se les documentó estenosis carotídea severa ( $70 \%-99 \%$ ), de los cuales $5 / 18$ sujetos (28 $\%)$ fueron llevados a endarterectomía. Todos los pacientes recibieron mejor tratamiento médico con terapia antiplaquetaria, estatina con terapia de alta intensidad y control de factores de riesgo, como lo sugiere la evidencia. La aspirina es el antiplaquetario más utilizado, seguro y efectivo administrado tempranamente $(<48 \mathrm{~h})$ posterior al evento vascular agudo; así mismo, el clopidogrel también ha tenido efectividad y reducción de riesgo de eventos vasculares. Adicionalmente, hay algunos estudios que sugieren mayor efectividad y reducción de riesgo de embolismo a corto plazo con antiagregación dual transitoria $(21,29)$.

Al comparar los dos periodos de estudios similares en el mismo hospital, se encontró una reducción ligera en la prevalencia de la enfermedad carotídea. También, menor porcentaje de fumadores en la última medición, lo que puede favorecer una reducción de la carga ateroesclerótica y ser resultado de las campañas antitabaco hechas por el Ministerio de Salud y Protección Social. De acuerdo con la etiología, en casi un $60 \%$ de los casos de estenosis extracraneal, esta explica los ACV de los pacientes. Hay competencia de etiologías para cardioembolismo en el $21 \%$ de los sujetos, y para enfermedad de pequeño vaso, en el $14 \%$. Esto representa que dentro de los que tienen afectación carotídea, cerca del $35 \%$ de los pacientes quedará como etiología criptogénica por la coexistencia de dos etiologías principales.

\section{Conclusiones}

Identificamos una prevalencia de enfermedad carotídea significativa (>50\%) del 12,3\%, similar a otros estudios, con una menor proporción en hombres, y se correlaciona de forma estrecha con los hallazgos del estudio previo del hospital (1996-2002) y con una mayor edad de presentación, entre 78 y 81 años, superior a otros estudios. La proporción de HTA y dislipidemia son preponderantes en esta patología, con tasas de diabetes, tabaquismo y enfermedad coronaria un poco menores respecto al estudio Nascet. Así mismo, un bajo porcentaje de pacientes fue llevado a intervenciones como endarterectomía o angioplastia, situación que amerita otro estudio clínico. 


\section{Limitaciones}

Este estudio incluye una muestra representativa de pacientes; sin embargo, al tratarse de un estudio observacional, tiene sesgos inherentes, como el de información, que se intentó minimizar con una revisión estricta de las historias clínicas (tomando aquellas con información completa), con bases de datos complementarias, con supervisión por parte de dos investigadores senior y con validación interna de los datos obtenidos.

\section{Conflicto de intereses}

Los autores declaramos no presentar conflictos de interés en la realización del presente trabajo de investigación

\section{Fuentes de financiación}

El trabajo fue hecho con recursos propios del Centro de ACV de la Fundación Santa Fe de Bogotá, además del trabajo voluntario de los investigadores.

\section{Agradecimientos}

A la doctora Ximena Varón, por facilitarnos los datos de su tesis de grado del 2002. Al doctor Éder Moreno, por la ayuda en el manejo de datos de la base del centro de ACV de la Fundación Santa Fe de Bogotá. Al Grupo de Investigación de Interés en Neurología, por el apoyo dado en las reuniones de seguimiento de proyectos y colaboración de estudiantes de pregrado. Al doctor Alberto Lineros, asesor metodológico de tesis de grado del 2020, base para la realización de este artículo, y a la Universidad El Bosque, institución que ha fomentado la investigación y especialización en el país.

\section{Referencias}

1. Guzik A, Bushnell C. Stroke epidemiology and risk factor management. Contin Neurol. 2017;23(1):15-39.

2. Christensen MC, Valiente R, Sampaio Silva G, Lee WC, Dutcher S, Guimarães Rocha MS, et al. Acute treatment costs of stroke in Brazil. Neuroepidemiology. 2009;32 (2):142-9. https://doi.org/10.1159/000184747

3. Moreno E, Rodríguez J, BayonaOrtiz H. Trombólisis endovenosa como tratamiento del $\mathrm{ACV}$ isquémico agudo en Colombia: una revisión sistemática de la literatura. Acta Neurol Colomb. 2019;35(3):156-66. https://doi.org/10. 22379/24224022262

4. De Weerd M, Greving JP, De Jong AWF, Buskens E, Bots ML. Prevalence of asymptomatic carotid artery stenosis according to age and sex systematic review and metaregression analysis. Stroke. 2009;40 (4):1105-13. https://doi.org/10 .1161/STROKEAHA.108.532218

5. Falk E. Pathogenesis of atherosclerosis. J Am Coll Cardiol. 2006;47 (8):7-12.

6. Badimon L, Vilahur G. Thrombosis formation on atherosclerotic lesions and plaque rupture. J Intern Med. 2014;276(6):618-32. https://doi.org/10 $.1111 /$ joim. 12296

7. Rothwell PM, Warlow CP. Low risk of ischemic stroke in patients with reduced internal carotid artery lumen diameter distal to severe symptomatic carotid stenosis: cerebral protection due to low poststenotic flow? Stroke. 2000;31(3):622-30.

8. Avezum Á, Costa-Filho FF, Pieri A, Martins SO, Marin-Neto JA. Stroke in Latin America: burden of disease and opportunities for prevention. Glob Heart. 2015;10(4):323-31. https://doi. org/10.1016/j.gheart.2014.01.006

9. Barnnet HJM, Taylor DW, Haynes RB. Beneficial effect 
Julieth Milena Rodríguez López, Carlos Fernando Martínez Rubio, Hernán Bayona Ortiz.

of carotid endarterectomy in symptomatic patients with high-grade carotid stenosis. $\mathrm{N}$ Engl J Med. $1991 ; 325(7): 445-53$.

10. American College of Cardiology Foundation/American Heart Association Task Force; American Stroke Association; American Association of Neuroscience Nurses; American Association of Neurological Surgeons; American College of Radiology; American Society of Neuroradiology; Congress of Neurological Surgeons; Society of Atherosclerosis Imaging and Prevention; Society for Cardiovascular Angiography and Interventions; Society of Interventional Radiology; Society of NeuroInterventional Surgery; Society for Vascular Medicine; Society for Vascular Surgery; American Academy of Neurology; Society of Cardiovascular Computed Tomography, Brott TG, Halperin JL, Abbara S, Bacharach JM, Barr JD, Bush RL, Cates CU, Creager MA, Fowler SB, Friday G, Hertzberg VS, McIff EB, Moore WS, Panagos PD, Riles TS, Rosenwasser RH, Taylor AJ. 2011 guideline on the management of patients with extracranial carotid and vertebral artery disease: Executive summary. J Neurointerv Surg. 2011;3(2):100-30. https://doi.org/10.1 136/jnis.2011.004762

11. Cosottini $\mathrm{M}$, Pingitore A, Puglioli M, Michelassi MC, Lupi G, Abbruzzese A, et al. Contrast-enhanced three-dimensional magnetic resonance angiography of atherosclerotic internal carotid stenosis as the noninvasive imaging modality in revascularization decision making. Stroke. 2003;34(3):660-4. https://doi.o rg/10.1161/01.STR.0000057462.0214 $1.6 \mathrm{~F}$
12. Wabnitz AM, Turan TN. Symptomatic carotid artery stenosis: Surgery, stenting, or medical therapy? Curr Treat Options Cardiovasc Med. 2017;19(8):1-12.

13. Amarenco P, Bogousslavsky J, Caplan LR, Donnan GA, Wolf ME, Hennerici MG. The ASCOD phenotyping of ischemic stroke (updated ASCO phenotyping). Cerebrovasc Dis. 2013;36(1):1-5. http s://doi.org/10.1159/000352050

14. Sacco RL, Kargman DE, Gu Q, Zamanillo MC. Race-ethnicity and determinants of intracranial atherosclerotic cerebral infarction. Stroke. 199526(1):14-20. https://doi.or g/10.1161/01.str.26.1.14

15. Strömberg S, Nordanstig A, Bentzel T, Österberg K, Bergström GM. Risk of early recurrent stroke in symptomatic carotid stenosis. Eur J Vasc Endovasc Surg. 2015;49(2):137-44. https://doi.or g/10.1016/j.ejvs.2014.11.004

16. Flaherty ML, Kissela B, Khoury JC, Alwell K, Moomaw CJ, Woo D, et al. Carotid artery stenosis as a cause of stroke. Neuroepidemiology. 2012;40(1):36-41. https://doi.org/10.1 $159 / 000341410$

17. Abbott AL, Bladin CF, Levi CR, Chambers BR. What should we do with asymptomatic carotid stenosis? Int J Stroke. 2007;2(1):27-39. https://doi.or g/10.1111/j.1747-4949.2007.00096.x

18. Spence JD. Asymptomatic carotid stenosis: why a moratorium is needed on intervention outside clinical trials. Neurology. 2017;88(21):1990-1.

19. Varón X, Bayona H. Prevalencia de enfermedad carotidea y relación con factores de riesgo en pacientes con ataque cerebrovascular. Bogotá: Universidad el Bosque, Posgrados; 2002. 
20. Nederkoorn PJ, Elgersma OEH, Mali WPTM, Eikelboom BC, Kappelle LJ, van der Graaf Y. Overestimation of carotid artery stenosis with magnetic resonance angiography compared with digital subtraction angiography. J Vasc Surg [internet]. 2002;36(4):806-13. Disponible en: https://www.jvascsurg.o $\mathrm{rg} /$ article/S0741-5214(02)00137-4/pdf 21. Lovrencic-Huzjan A, Rundek T, Katsnelson M. Recommendations for management of patients with carotid stenosis. Stroke Res Treat. 2012;2012:1-12.

22. Ong C-T, Wong Y-S, Sung S-F, Wu $\mathrm{CS}, \mathrm{Hsu} Y \mathrm{YC}, \mathrm{Su} \mathrm{YH}$, et al. Progression of mild to moderate stenosis in the internal carotid arteries of patients with ischemic stroke. Front Neurol. 2018;9:1-7. https://doi.org/10.3389/fne ur.2018.01043

23. Joh JH. The prevalence and risk factors for atherosclerotic carotid stenosis and carotid plaque: A community-based screening study. J Vasc Surg. 2015;61(6):91S-92S.

24. Woo SY, Joh JH, Han SA, Park HC. Prevalence and risk factors for atherosclerotic carotid stenosis and plaque a population-based screening study. Medicine (Baltimore). 2017;96(4):2-7. https://doi.org/10.109 7/MD.0000000000005999

25. Barnett H, Guton RW, Eliasziw M, Stern BJ, George MG; WASID Trial Investigators. Causes and severity of ischemic stroke in patients with symptomatic intracranial arterial stenosis. Stroke. 2009;40(6):1999-2003. https://doi.org/ 10.1161/STROKEAHA.108.546150

26. Barnett HJ, Taylor DW, Eliasziw M, Fox AJ, Ferguson GG, Haynes RB, et al. Benefit of carotid endarterectomy in patients with symptomatic moderate or severe stenosis. $\mathrm{N}$ Engl J Med.
1998;339(20):1415-25. https://doi.org/ 10.1056/NEJM199811123392002

27. Turunen MP, Hiltunen MO, Ylä-Herttuala S. Gene therapy for angiogenesis, restenosis and related diseases. Exp Gerontol. 1999;34(4):567-74.

28. Amarenco P, Kim JS, Labreuche J, Charles H, Abtan J, Béjot Y, et al. A comparison of two LDL cholesterol targets after ischemic stroke. $\mathrm{N}$ Engl J Med. 2020;382(1):9-19. https://doi.org /10.1056/NEJMoa1910355

29. Markus HS, Droste DW, Kaps M, Larrue V, Lees KR, Siebler M, et al. Dual antiplatelet therapy with clopidogrel and aspirin in symptomatic carotid stenosis evaluated using doppler embolic signal detection: The clopidogrel and aspirin for reduction of emboli in symptomatic carotid stenosis (CARESS) trial. Circulation. 2005;111(17):2233-40. https://doi.org/ 10.1161/01.CIR.0000163561.90680.1 C 